\title{
A REMARK ON KERNELS OF REDUCTION
}

\author{
ROBERT F. COLEMAN
}

(Communicated by Louis J. Ratliff, Jr.)

\begin{abstract}
We show the group of torsion points on an Abelian variety defined over an algebraic closure of the rationals, $\overline{\mathbf{Q}}$ is generated by the kernels of reduction of all the primes of $\overline{\mathbf{Q}}$.
\end{abstract}

Let $A$ be an Abelian variety over $\overline{\mathbf{Q}}$. For each prime $\mu$ of $\overline{\mathbf{Q}}$ let $K_{\mu}$ denote the kernel of reduction in $A(\overline{\mathbf{Q}})$ at $\mu$. This makes sense even at primes of bad reduction since $A$ has a unique semi-Abelian model over the ring of integers in $\overline{\mathbf{Q}}$. For each integer $n$, let $A[n]$ denote the kernel of multiplication by $n$ in $A(\overline{\mathbf{Q}})$ and $K_{n}=K_{A, n}$ the smallest subgroup of $A[n]$ containing $A[n] \cap K_{\mu}$ for all primes $\rho$ of $\mathbf{Q}$. If $A$ is defined over a number field $F$, then so is $K_{n}$.

Our goal in this note is to prove

THEOREM A. The index of $K_{n}$ in $A[n]$ is bounded independently of $n$.

We will need the following

THEOREM B (FALTINGS AND ZARHIN [F], [Z]). Let $F$ be a number field. Then in each isogency class of Abelian varieties over $F$ there are finitely many isomorphism classes.

We will also need the following lemmas:

LEMMA C. Suppose $A$ is a semi-Abelian variety over the ring of integers in a number field $F$ whose generic fiber is Abelian. Suppose $b$ is an endomorphism of $A$ and $p$ is a prime number which divides the degree of $b$. Then $b$ has inseparable reduction at some prime above $p$.

ProOF. Let $\Omega$ denote the module of invariant differentials on $A$ and $\Lambda$ its maximal exterior power. Let $\beta$ denote the eigenvalue of $b$ on $\Lambda$. We see that $\beta \bar{\beta}=\operatorname{det} b$ for any complex conjugation " $-"$. It follows that there exists a prime $\rho$ above $p$ dividing $\beta$ and $b$ has inseparable reduction at $\rho$.

LEMMA D. Supose $A$ and $B$ are semi-Abelian varieties over the ring of integers in a finite extension of $\mathbf{Q}_{p}$ whose generic fibers are Abelian. Suppose $a: A \rightarrow B$ and $b: B \rightarrow A$ are isogenies such that $b \circ a=d p^{m}$, where $(d, p)=1$ and the kernel of $a$ contains the kernel of reduction inside $A\left[p^{m}\right]$. Then the reduction of $b$ is separable.

PROOF. This follows immediately from the fact that the subgroup of the kernel of $b$ lying on the connected component of $B$ injects into the reduction of $B$.

Let $A_{n}=A / K_{n}$. Let $a_{n}: A \rightarrow A_{n}$ denote the natural isogeny and $a_{n}^{\prime}: A_{n} \rightarrow A$ the isogeny such that $a_{n}^{\prime} \circ a_{n}=n$.

Received by the editors October 5, 1987 and, in revised form, November 20, 1987.

1980 Mathematics Subject Classification (1985 Revision). Primary 11G10; Secondary 14K19. 
LemMA E. Suppose $d m=n$. Let $B=A_{d}$; then $K_{B, m}=a_{d}\left(K_{n}\right)$.

PrOOF. Clearly

$$
K_{B, m}=a_{d}\left(\sum\left\{x \in A(\overline{\mathbf{Q}}): m x \in K_{d}, x_{\mu} \in\left(K_{d}\right)_{\mu}\right\}\right),
$$

where the sum runs over all primes $\rho$ of $\overline{\mathbf{Q}}$ and the subscript $\rho$ denotes reduction modulo $\mu$. Now $\left\{x \in A(\overline{\mathbf{Q}}): m x \in K_{d}, x_{\rho} \in\left(K_{d}\right)_{\rho}\right\}$ equals

$\left\{x \in A(\overline{\mathbf{Q}}): n x=0, \exists y \in K_{d}\right.$ such that $\left.(x-y) \in K_{\mu}\right\}=K_{d}+\left(A[n] \cap K_{\mu}\right)$.

Since $K_{d} \subseteq K_{n}$ it follows that $K_{B, m}=a_{d}\left(K_{n}\right)$.

COROLlaRY. If $d n=m$, then $\left[A[d]: K_{d}\right]$ divides $\left[A[n]: K_{n}\right]$. If $(d, m)=1$, then $\left[A[n]: K_{n}\right]=\left[A[d]: K_{d}\right] \cdot\left[A[m]: K_{m}\right]$.

PrOOF. We have immediately from the lemma

$$
\left[B[m]: K_{B, m}\right]=\left[A[n]: K_{n}\right] /\left[A[d]: K_{d}\right] .
$$

This implies the first part. The second part follows from the fact that $K_{n}=K_{m} \cdot K_{d}$ when $(m, d)=1$.

PROPOSITION F. Suppose $m$ and $n$ are integers such that

$$
\left[A[m]: K_{m}\right] \neq\left[A[n]: K_{n}\right],
$$

then $A_{m}$ and $A_{n}$ are not isomorphic.

PrOOF. Suppose $p$ is a prime such that

$$
\operatorname{ord}_{p}\left[A[n]: K_{n}\right]>\operatorname{ord}_{p}\left[A[m]: K_{m}\right]
$$

and $A_{m}$ and $A_{n}$ are isomorphic. We may suppose that $A$ is defined and has a semi-Abelian model over the ring of integers in a finite extension $F$ of $\mathbf{Q}$. We may suppose, in addition, that there exists an isomorphism $\iota: A_{n} \rightarrow A_{m}$ defined over $F$.

Let $k=\operatorname{ord}_{p} m$. It follows from the previous Corollary that $p^{k}$ divides $n$. Hence, by Lemma E, we may replace $A$ with $A_{p^{k}}$ and suppose that $p$ does not divide $m$ and hence does not divide $\left[A[m]: K_{m}\right]$.

Let $a$ denote the endomorphism of $A, a_{m}^{\prime} \circ \iota \circ a_{n}$. Then Ker $a \supseteq K_{n}$ and there exists an endomorphism $b$ of $A$ such that $a \circ b=m n$. Since $(m, p)=1$ it follows from Lemma $\mathrm{D}$ that the reduction of $b$ (with respect to the semi-Abelian model) is separable at all primes dividing $p$. But the hypotheses imply that $p$ divides the degree of $b$. This contradicts Lemma $\mathrm{C}$ and proves the Proposition.

PROOF OF THEOREM A. We may assume without loss of generality that $A$ is defined over a finite extension $F$ of $\overline{\mathbf{Q}}$ and has a semi-Abelian model over the ring of integers of $F$. Then $A_{n}$ and $a_{n}$ are defined over $F$.

Since, all the $A_{n}$ are isogenous, they lie in finitely many isomorphism classes over $F$. It follows from Proposition $\mathrm{F}$ that the set of integers $\left\{\left[A[n]: K_{n}\right]: n \in \mathrm{N}\right\}$ is finite. Theorem A follows immediately.

REMARK. Let $A$ denote the elliptic curve $y^{2}=x(x-1)(x-2)$. Then $\left[A[2]: K_{2}\right]$ $=2$. 


\section{REFERENCES}

[F] G. Faltings, Enlicheitsätz für Abelsche Varietäten über Zahlkörpern, Invent. Math. 74 (1983), 349-366.

[Z] Yu G. Zarhin, A finiteness theorem for unpolarized Abelian varieties over number fields with prescribed places of bad reduction, Invent. Math. 79 (1985), 309-321.

Department of MAThematics, UNiversity of CALIFornia, Berkeley, CALIFORNIA 94530 